\title{
COVID-19 in humanitarian settings and lessons learned from past epidemics
}

\author{
In the COVID-19 pandemic, the most vulnerable people are most likely to be the hardest hit. What can we learn \\ from past epidemics to protect not only refugees but also the wider population?
}

\author{
Ling San Lau, Goleen Samari, Rachel T. Moresky, Sara E. Casey, S. Patrick Kachur, Leslie F. Roberts and \\ Monette Zard
}

$\Lambda$ lthough World Health Organization Director-General Tedros Adhanom Ghebreyesus has urged a "whole-ofgovernment, whole-of-society approach" to COVID-19, displaced populations, including refugees, have been stigmatized, scapegoated and neglected in the emergency response. These populations confront substantial vulnerabilities in the context of COVID-19. They often experience substandard living conditions, overcrowding, limited access to safe water and sanitation, and poor health and nutrition, thus substantially increasing their risk of infection ${ }^{1}$. In addition, they may face greater difficulties than the general population in accessing health services ${ }^{1}$ and may disproportionately bear the burden of pandemic-control measures, including restrictions on movement and border closures. In humanitarian contexts, conflict, political instability, resource limitations, poor governance, and weak health systems and public-health infrastructures further constrain the ability to detect and respond effectively to outbreaks ${ }^{2}$.

According to UNHCR, the UN Refugee Agency, of the 196 countries affected by COVID-19 globally, 79 are refugee-hosting countries reporting local transmission ${ }^{3}$. COVID-19 will inevitably spread to displaced communities, and we urgently need to act to mitigate the short-term and lasting effects on displaced populations and the wider community. Our experience with past epidemics and pandemics, including Ebola, severe acute respiratory syndrome (SARS) and Middle East respiratory syndrome, provides important lessons about what to expect and prepare for as we navigate the challenges ahead.

\footnotetext{
Maintaining essential health services is vital to reduce additional deaths from COVID-19

We know from previous epidemics, including Ebola and H1N1 influenza, that indirect health effects exceed the deaths and morbidity caused by the disease. During a pandemic response, the diversion of
}

resources, disruptions to medical supply chains, health-facility closures and shortages of healthcare workers (due to deaths, illness, absenteeism, care responsibilities or control measures) can overwhelm health systems, particularly in humanitarian contexts. These restrictions can exacerbate existing disparities in resource distribution between urban and rural areas and informal settlements. Restrictions on movement, legal-status concerns and fears of contagion may also reduce individuals' ability or willingness to access health care.

People with chronic conditions, including HIV, tuberculosis, cardiovascular disease and diabetes, require continuity of care and are at increased risk of death or complications if their treatment is interrupted ${ }^{4,5}$. They are also more susceptible to severe and fatal COVID-19 infection. The humanitarian system is already poorly equipped to manage chronic-disease needs ${ }^{6}$. During the 2014-2016 Ebola epidemic in West Africa, reduced access to treatment for malaria, HIV/AIDS and tuberculosis was estimated to cause 10,600 excess deaths in Guinea, Liberia and Sierra Leone ${ }^{7}$, a number rivaling the total 11,300 Ebola deaths reported in these countries. Other studies have found significant decreases in routine visits for maternal and child health, reproductive health, and common childhood illnesses in Ebola-affected countries $^{8,9}$. Studies have shown that even in well-resourced settings, increased healthcare burdens due to the $2009 \mathrm{H} 1 \mathrm{~N} 1$ influenza pandemic were associated with increased deaths from stroke, acute myocardial infarction and congestive heart failure ${ }^{10}$. As the international community ramps up its response to COVID-19, ensuring the continuity of essential health services and prevention programs that are necessary to save lives is imperative. Doing so requires immediate attention to the health needs and priorities of displaced populations and contingency planning to ensure service continuity. Possible approaches include alternative treatment-delivery methods, supply-chain management and the provision of extended medication supplies for conditions including HIV, tuberculosis and non-communicable diseases, as well as modern contraceptive methods ${ }^{11,12}$. Protecting healthcare workers, ensuring that they feel safe to continue working (including adequate provision of personal protective equipment, supportive supervision and continuous pay) and not unduly restricting their ability to work are also essential.

\section{Access to infection prevention and} control measures, testing and treatment for displaced populations is essential to ensure safety for all Ensuring that displaced populations, as well as the healthcare workers who serve them, have access to infection prevention and control measures, testing and treatment is important. As critical shortages in personal protective equipment, testing resources and ventilators are being reported worldwide, advocating for the needs of displaced populations and people working with them is essential. Although displaced populations should be integrated into national health-response processes, many countries appear to be prioritizing the needs of citizens within their borders. Refugees in a camp in Lesbos, Greece, have resorted to sewing handmade face masks for residents who may fall ill ${ }^{13}$. Social distancing is important to reduce the rate of community transmission, yet it is a luxury rarely available to displaced people living in camps, detention facilities, high-density urban areas, or informal settlements. These sites are densely populated, often lack adequate water and sanitation, and feature shared latrines and cooking facilities. Further, humanitarian-service provision, including food distribution, typically requires people to assemble in crowds.

Interim guidance from the Inter-Agency Standing Committee (IASC) on COVID-19 outbreak readiness and response in humanitarian situations advises strategic site planning to enable better distancing, 
attention to crowd management, and strengthened surveillance and testing to detect initial cases early ${ }^{1}$. For severe cases, displaced populations need access to hospitals with intensive care unit beds and ventilators. Special attention must be paid to the subgroups most vulnerable to COVID-19, including individuals who are over 50 years old, are immunocompromised or are living with non-communicable diseases such as cardiovascular disease, respiratory disease or diabetes. These groups in particular require the ability to social distance, as well as access to proper handhygiene training and supplies, and free COVID-19 testing and treatment.

\section{Pandemic control relies on community engagement and trust}

Our experience with Ebola and SARS demonstrates that transparency, trust and community partnership are key to efforts for controlling COVID-19. Lessons learned from SARS show the importance of early detection, risk communication with vulnerable groups, and strategies for containment and multinational collaboration $^{14}$. Effective communication and public trust are crucial to the success of public-health measures, as they require the cooperation of affected individuals and communities. Communication and trust are important for displaced populations, which, as a result of previous experiences, may lack trust in the authorities or systems that are supposed to serve their needs ${ }^{15}$.

Although travel restrictions have been applied globally in response to COVID-19, the World Health Organization has counseled against broad restrictions that fuel fear, impede effective transfer of medical personnel and supplies, and almost always come too late to contain the spread of the disease $\mathrm{e}^{16}$. Restrictions on freedom of movement must not be applied in a discriminatory way to displaced populations, and the principle of non-refoulement must be respected regardless of real or perceived fears of coronavirus ${ }^{17}$. Travel bans, when combined with populist rhetoric that seeks to blame the virus on 'foreigners', can incite stigma and scapegoat migrant communities, thus undermining public-health goals. In contrast, engaging affected communities and trusted sources, including survivors and community leaders, can contribute to the overall success of health-related security efforts worldwide ${ }^{18}$. There is a role for safe quarantine and isolation in combating COVID-19, but these measures must be thoughtfully applied with respect for rights and specific circumstances ${ }^{15}$. In humanitarian settings, quarantine can be challenging, and efforts must be made to ensure that quarantined individuals can continue to have access to basic needs, food and support services ${ }^{15}$. Moreover, displaced populations must critically receive clear messages about the role and importance of quarantine as a means of containing infectious diseases throughout the course of the response.

\section{Conclusion}

The rapid global spread of a novel coronavirus has taught us that we are all interconnected in terms of our health and well-being. Displaced populations, including refugees and migrants, are often the first to be stigmatized and unjustly blamed for the spread of disease, yet they are also among the most vulnerable people during a pandemic - to both the virus itself and the measures enacted to control it. Substantial disruptions to humanitarian operations have already been reported from the field, thus compromising access to health and other essential services, and the ability to respond to urgent community needs. In the words of one humanitarian worker: "COVID-19 is making the already vulnerable exponentially more vulnerable, and it hasn't even hit yet."

We must apply the lessons of past pandemics in our response to COVID-19, including paying heed to the indirect health effects of the disease, to reduce mortality, and ensuring that refugees and displaced populations have access to testing, referral and definitive treatment alongside all populations. Previous epidemics have shown us that stigmatizing viral transmission only further places populations at risk and reduces access to care ${ }^{19}$. Harsh migration enforcement and disregard for displaced populations undermines the first defense that we have in public health, which is the willingness of people to disclose symptoms and seek care. Trust is a critical commodity in pandemic responses, and it must be preserved. Pandemics do not discriminate, and access to health care should not discriminate either. In the age of COVID-19, protecting the most vulnerable among us is not just a moral imperative but an urgent public health objective: the health of one is the health of all.

Ling San Lau', Goleen Samari',

Rachel T. Moresky1,2, Sara E. Casey (D)1,

S. Patrick Kachur (D', Leslie F. Roberts ${ }^{1}$ and Monette Zard ${ }^{1 \times}$

${ }^{1}$ Program on Forced Migration and Health, Heilbrunn Department of Population and Family Health, Mailman School of Public Health, Columbia University, New York, NY, USA. ${ }^{2}$ Department of Emergency Medicine, Irving Medical Center, Columbia University, New York, NY, USA.

$\bigotimes_{\text {e-mail: mz2621@cumc.columbia.edu }}$

Published online: 8 April 2020

https://doi.org/10.1038/s41591-020-0851-2

References

1. IFRC. IOM, UNHCR, WHO. Scaling-Up Covid-19 Outbreak Readiness and Response Operations in Humanitarian Situations (Inter-Agency Standing Committee Secretariat, 2020).

2. Madhav, N. et al. in Disease Control Priorities: Improving Health and Reducing Poverty (eds Jamison, D. T. et al.) (World Bank, 2017).

3. UNHCR. Coronavirus Emergency Appeal: UNHCR's Preparedness and Response Plan (revised) (30 March 2020).

4. Konwloh, P. K. et al. Public Health Action 7(Suppl. 1), S62-S69 (2017).

5. Hussey, P. S. et al. JAMA Intern. Med. 174, 742 (2014).

6. Coutts, A. et al. Lancet Respir. Med. 3, e8-e9 (2015).

7. Parpia, A. S., Ndeffo-Mbah, M. L., Wenzel, N. S. \& Galvani, A. P. Emerg. Infect. Dis. 22, 433-441 (2016).

8. Barden-O'Fallon, J., Barry, M.A., Brodish, P. \& Hazerjian, J. PLoS Curr. 7, ecurrents.outbreaks.0b0ba06009dd091bc39ddb3c 6d7b0826 (2015).

9. Assessing the Socio-Economic Impacts of Ebola Virus Disease in Guinea, Liberia, and Sierra Leone: The Road to Recovery (United Nations Development Program, 2014).

10. Rubinson, L. et al. Med. Care 51, 259-265 (2013).

11. Bamrah, S. et al. Prehosp. Disaster Med. 28, 43-51 (2013).

12. Heffelfinger, J. D. et al. Am. J. Public Health 99(Suppl. 2), S333-S339 (2009)

13. Fallon, K. The Greek refugees battling to prevent Covid-19 with handmade face masks. The Guardian (18 March 2020).

14. Oberholtzer, K. et al. Learning from SARS: Preparing for the Next Disease Outbreak: Workshop Summary (National Academies Press, 2004).

15. Vaughan, E. \& Tinker, T. Am. J. Public Health 99(Suppl. 2), S324-S332 (2009)

16. Mateus, A. L., Otete, H. E., Beck, C. R., Dolan, G. P. \& Nguyen-Van-Tam, J. S. Bull. World Health Organ. 92, 868-880D (2014).

17. Høvring, R. 10 things you should know about coronivirus and refugees. Norwegian Refugee Council https://www.nrc. no/news/2020/march/10-things-you-should-know-aboutcoronavirus-and-refugees/ (16 March, 2020)

18. Dahn, B., Fallah, M. P., Platts, J., Moon, S. \& Kimball, A. M. Lancet 389, 1189 (2017).

19. Person, B. et al. Emerg. Infect. Dis. 10, 358-363 (2004).

Acknowledgements

We thank S. Guyer for research assistance in compiling this Comment.

Competing interests

The authors declare no competing interests. 